\title{
FMO superfamily protein phylogeny and the origin of YUCCA family
}

\author{
Igor Turnaev \\ Systems Biology Department \\ ICG SB RAS, Novosibirsk, Russia \\ turn@bionen.nsc.ru
}

\author{
Valentin Suslov \\ Systems Biology Department \\ ICG SB RAS, Novosibirsk, Russia \\ valya@bionen.nsc.ru \\ Konstantin Gunbin \\ Systems Biology Department \\ ICG SB RAS, Novosibirsk, Russia \\ genkvg@bionen.nsc.ru
}

\author{
Dmitriy Afonnikov \\ Systems Biology Department \\ ICG SB RAS, Novosibirsk, Russia \\ ada@bionen.nsc.ru
}

\begin{abstract}
We performed a comparative analysis of phylogeny, conservative sites, protein domains, and the presence in taxa of FMOs and BVMOs proteins. Earlier in the FMOs, the following protein groups were distinguished: type I FMO, type II FMO, YUCCAs. According to the results of our analysis, we propose that the type II FMO group is, in turn, divided into two groups: FMO IIA type and type IIB FMO. Type IIB FMO proteins represent a new special group in a number of parameters (site consensus, composition of protein domains, presence in taxa and, apparently, functions) significantly different from all other FMOs proteins and type IIA FMO proteins, in particular. While type IIA FMO proteins exhibit properties typical of the FMO subclass. The type IIB FMO protein group is likely to represent a new family of proteins. These proteins are of interest for biocatalysis.
\end{abstract}

Keywords - protein phylogeny; protein evolution; flavin monooxygenase; auxin biosynthesis; YUCCA family; conservative motifs

\section{Background}

YUCCA proteins are important plant enzymes involved in the biosynthesis of one of the key hormones, auxin. [1]. It is believed that these genes originated as a result of horizontal transfer from bacteria to plant genome [2].

YUCCA enzymes belong to the superfamily of flavindependent monooxygenases (FMO), therefore, studying the position of the proteins of the YUCCA family within the FMO superfamily can shed light on the origin of the auxin biosynthesis pathway in higher plants.

Class B flavin-dependent monooxygenases (EC 1.14.13) include three subclasses: N-hydroxylating monooxygenases (NMOs), Baeyer - Villiger monooxygenases (BVMOs), and flavin-containing monooxygenases (FMOs). The YUCCA protein family belongs to the last subclass of FMOs [3].

In this work, a detailed analysis of the phylogeny of FMOs proteins was carried out, which allowed us to make an assumption that there are two subgroups, type IIA and IIB, in the type II FMO group. Subgroup IIA, but not IIB, is sister to YUCCA. Based on these data, assumptions were made about the origin and evolution of the proteins of the YUCCA family.

\section{Materials and methods}

Proteins of YUCCAs family and their closest homologues were extracted from NCBI database using protein BLAST with a threshold e-value $<1 \mathrm{e}-5$. Homologs of protein YUC2 (AT4G13260) of Arabidopsis thaliana were the BLAST queries; only full-size proteins were taking into account. Promals used for alignment reconstruction. The phylogenetic tree was built using Maximum Likelihood methods. Protein motifs were built using WebLogo (HTTPS://WEBLOGO.BERKELEY.EDU).

\section{Results and Discussions}

We have built a FMO-like (YUCs homologues) phylogenetic tree (as a target for the search was taken protein YUC2 Arabidopsis thaliana - At4G13260, e-value $\leq 1 \mathrm{e}-05$ ) (fig. 1). In addition to FMO proteins, Baeyer-Villiger monooxygenases (BVMO) were taken as outgroup.

In the phylogenetic tree, we have identified 5 groups of proteins: (1) YUCCAs; (2) type II FMO (on the basis of Riebel's FMO classification - [4]); (3) type I FMO (on the basis of Riebel's FMO classification); (4) NMOs; (5) BVMOs. According to the results of the analysis group (2) - type II FMO is divided into 2 subclusters, which we called type IIA FMO and type IIB FMO.

Comparative analysis of conservative motifs among groups of FMO proteins.

All proteins of type B flavin monooxygenases (FMOs, NMOs and BVMOs) contain three conservative sites (fig. 1A). Two typical Rossman fold motives (GxGxxG/A): the first is FAD binding site located closer to the $\mathrm{N}$-terminus and the second is the NADPH binding site near the middle of the protein $[4,5]$. Between them is the FMO identification site (FxGxxxHxxxF/Y/W).

In the sequences of the FMOs subclass, the last amino acid at this site is $\mathrm{F} / \mathrm{Y}$, and that of the BVMO subclass is W. NMOs has lost its identification site, leaving only one central histiditis (xxxxxxHxxxx) [6]. In this connection, NMOs proteins are not included in further analysis.

A)

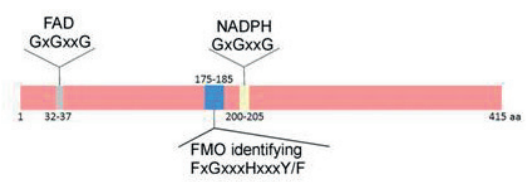


B)

\begin{tabular}{|c|c|c|c|}
\hline $\begin{array}{l}\text { Conservative sites } \\
\text { names }\end{array}$ & FAD binding site & FAD identified site & $\begin{array}{l}\text { NADPH } \\
\text { binding site }\end{array}$ \\
\hline Site motives & GxGxxG & $\operatorname{FxGxxxHxxx^{\mathrm {Y}}/\mathrm {F}/\mathrm {K}}$ & GxGxxG/A \\
\hline i. YUCCA & & 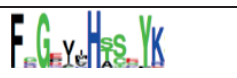 & GCENSC \\
\hline ii. type IIA FMO & GAfraf & 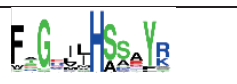 & G_GNGG \\
\hline iii. type IIB FMO & & 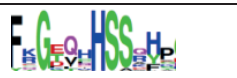 & GSNNSA \\
\hline iv. type I FMO & $G A G=S$ & 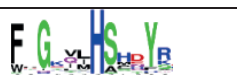 & G.GSA \\
\hline v. BVMOs & GafFsG & 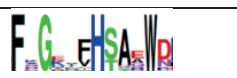 & GTGASA \\
\hline
\end{tabular}

Fig. 1. Distribution and alignment of key functional sites of FMOs / BVMOs homolog proteins. A) The localization of motifs along FMO proteins is shown schematically (the amino acid positions in the diagram are indicated by the sequence AT4G13260 - Arabidopsis thaliana YUC2). B) The table, respectively, in columns 2-4 shows the consensus of the three motives in FMOs / BVMOs proteins: FMO-binding, FMO identifying and NADH-binding sites.

We analyzed the sequences of three functional motifs in five protein clusters isolated on the phylogenetic tree: YUCCAs, type IIA FMO, type IIB FMO, type I FMO, BVMOs (fig. 1B). The motives for these three sites were built using WebLogo. The first motive is the FAD binding site - the motif for the sequences of all protein groups is similar and contains 3 highly conserved glycines. The most interesting are the cardinal differences between the sequences on the one hand, type IIB FMO, and on the other, type I, IIA, IIC FMOs, YUCCAs in the second - FMO identification site - and in the third - NADPH binding site motives. So, in type IIB FMO sequences, in the FMO identified motif, in $70 \%$ of cases, instead of $\mathrm{Y} / \mathrm{F}$, histidine $(\mathrm{H})$ is located, and in the NADPH motif, in the third position, in $75 \%$ case, instead of glycine $(\mathrm{G})$ is asparagine $(\mathrm{N})$. This suggests that type FMO IIB proteins may, like BVMO proteins, belong to a family separate from other FMOs that perform other functions than YUCCAs proteins, type I FMO and type IIA FMO.

\section{Comparative analysis of domains of FMO proteins}

The analysis of FMO and BVMO protein domains showed that type FMO IIB proteins contain an N-terminal domain of about 160 aa. not characteristic of either FMO-like (YUCs, type I FMO, type FMO IIA), nor for BVMO proteins.

\section{Characteristic group of proteins type IIB FMO}

The type FMO IIB includes proteins of bacteria, fungi, charophyte Klebsormidium nitens GAQ32387 protein and 4 proteins from club moss Selaginella moellendorffii. Previously identified by the group Wang et. al. [7] homology of GAQ32387 $K$. nitens protein with land plant YUCs proteins allowed them to hypothesize the earlier origin (TAA-YUCCA) of the auxin biosynthesis pathway already in charophytes, and not in the first land plant, as previously thought [2]. According to our data, GAQ32387 refers to type IIB FMO. An additional search for
GAQ32387 homologs among the RNAseq sequences of the base 1000 plant genomes revealed its close homologues among the red algae, lower green algae, moss, club moss, ferns, gymnosperms, monocots and dicots. This implies a significantly wider distribution of type FMO IIB proteins in plants than could be expected.

\section{Conclusions}

1) According to the results of our analysis, we propose the group protein type II FMO (according to the classification Riebel et al.'s [4]) divided into two groups type FMO IIA and type FMO IIB; 2) The type FMO IIB proteins seem to possess properties unique to FMO and BVMO (fig. 5), as has already been shown for proteins FMOe, f, g R. jostii RHA1 [8]; 3) The remoteness of type FMO IIB proteins and, accordingly, GAQ32383 K. nitens protein from YUCs on phylogeny and their structural and probably functional uniqueness is in favor of the origin of YUCs in first land plants (Yue et. al.'s [2] hypothesis), and not in Charophyceae (Wang et. al.'s [Wang et. al., 2014 7] hypothesis).

\section{Acknowledgment}

The work supported by the budget project No. 0324-20190040-C-01.

\section{References}

[1] K. Mashiguchi, K. Tanaka, T. Sakai, S. Sugawara, H. Kawaide, M. Natsume et al., "The main auxin biosynthesis pathway in Arabidopsis.” Proc. Natl. Acad. Sci. U S A, vol. 108(45), pp. 1851218517, 2011.

[2] Yue J., X. Hu, and J. Huang, "Origin of plant auxin biosynthesis." Trends in Plant Science, vol. 19(12), pp. 764-770, 2014.

[3] M. M. E. Huijbers, S. Montersino, A. H. Westphal, D. Tischler, W. J. H. van Berkel, "Flavin dependent monooxygenases." Archives of Biochemistry and Biophysics, vol. 544, pp. 2-17, 2014.

[4] A. Riebel, G. de Gonzalo, M. F. Fraaije, "In Expanding the biocatalytic toolbox of flavoprotein monooxygenases from Rhodococcus jostii RHA1." Enzymatic, vol. 88, pp. 20- 25, 2013.

[5] A. Riebel, H. M. Dudek, G. de Gonzalo, P. Stepniak, L. Rychlewski, M. W. Fraaije, "Expanding the set of rhodococcal Baeyer-Villiger monooxygenases by high-throughput cloning, expression and substrate screening. Appl Microbiol Biotechnol, vol. 95(6), pp. 14791489, 2012.

[6] M. W. Fraaije, N. M. Kamerbeek, W. J van Berkel, D.B Janssen, "Identification of a Baeyer-Villiger monooxygenase sequence motif.” FEBS Lett., vol. 518(1-3), pp. 43-47, 2002.

[7] C. Wang, Y. Liu, S.-S. Li and G.-Z. Han, "Origin of plant auxin biosynthesis in charophyte algae." Trends in Plant Science, vol. 19(12), pp. 741-743, 2014.

[8] A. Riebel, M. J. Fink, M. D. Mihovilovic, M. W. Fraaije, "Type II Flavin-Containing Monooxygenases: A New Class of Biocatalysts that Harbors Baeyer-Villiger Monooxygenases with a Relaxed Coenzyme Specificity." ChemCatChem, 2014, vol. 6(4), pp. 11121117, 2014. 